\title{
The effects of locations on the build tray on the quality of specimens in powder bed additive manufacturing
}

\author{
Snehashis Pal ${ }^{1}$ (D) Nenad Gubeljak ${ }^{1} \cdot$ Tonica Bončina $^{1} \cdot$ Radovan Hudák $^{2} \cdot$ Teodor Toth $^{2} \cdot$ Jozef Zivcak $^{2}$ • \\ Gorazd Lojen $^{1} \cdot$ Niko Leben ${ }^{3} \cdot$ Igor Drstvenšek ${ }^{1}$
}

Received: 22 July 2020 / Accepted: 21 December 2020 / Published online: 2 January 2021

(C) The Author(s) 2021

\begin{abstract}
In this study, the effect of powder spreading direction was investigated on selectively laser-melted specimens. The results showed that the metallurgical properties of the specimens varied during fabrication with respect to their position on the build tray. The density, porosity, and tensile properties of the $\mathrm{Co}-\mathrm{Cr}-\mathrm{W}-\mathrm{Mo}$ alloy were investigated on cuboid and tensile specimens fabricated at different locations. Two different significant positions on the tray were selected along the powder spreading direction. One set of specimens was located near the start line of powder spreading, and the other set was located near the end of the building tray. The main role in the consequences of powder layering was played by the distribution of powder particle sizes and the packing density of the layers. As a result, laser penetration, melt pool formation, and fusion characteristics varied. To confirm the occurrence of variations in sample density, an additional experiment was performed with a Ti-6Al-4V alloy. Furthermore, the powders were collected at two different fabricating locations and their size distribution for both materials was investigated.
\end{abstract}

Keywords Fabrication location $\cdot$ Powder coating direction $\cdot$ Density $\cdot$ Porosity $\cdot$ Tensile properties $\cdot$ Selective laser melting

\section{Introduction}

Several studies have been carried out on the properties of products made with the powder bed additive manufacturing processes, in particular the selective laser melting (SLM) process $[1-3]$. However, these investigations were based on employing different processing parameters [4-6], scanning strategies [7], and different build-up directions due to different angular orientations of the part $[8,9]$. Eventually, limited research was carried out focusing on the effect of build-up locations by means of the powder spreading direction. Due to the various applications of SLM products in several important fields, such as aerospace, automotive and biomedical implants, sublime quality, and appropriateness are the most

Snehashis Pal

snehashiseu@gmail.com

1 Faculty of Mechanical Engineering, University of Maribor, Smetanova ulica 17, 2000 Maribor, Slovenia

2 Department of Biomedical Engineering and Measurement, Technical University of Kosice, Kosice, Slovakia

3 Dentas L.L.C., Cesta k Tamu 70, 2000 Maribor, Slovenia important requirements $[10,11]$. The choice of the SLM fabrication process for customizing complex products is becoming higher due to its high manufacturing flexibility, with lower cost and in less time compared to traditional manufacturing methods [12].

In the SLM process, metal powders are deposited in layers, then melted and fused together with a laser to obtain a solid part, as entrusted computer-aided design model [13]. A powder coating system, also known as re-coater or a powder spreading blade, distributes the powder particles onto the building platform using a raking mechanism known as respreading [14]. The powder re-coater collects the powder from the supply chamber, spreads it over the building tray, and conveys the excess powder into the overflow chamber [15]. A limited amount of powder can be carried by the powder re-coater due to the few limitations imposed by surplus powder availability, the process chamber volume, the design of the machine design, etc. The powder is spread by a raking mechanism utilizing the weight force of the powder accumulated by the re-coater over the intended powder layer. The combined weight force acting through the powder particles above the powder layer decreases as the powder is spread behind the re-coater. Hence, it may happen that the powder is unevenly distributed across the build tray from the starting 
to the end line of spreading. Moreover, the size of the powder particles is in the range from a few micrometers to a few tens of micrometers $(10-45 \mu \mathrm{m}$ according to the producer's datasheets). On their way from the powder feed chamber to the overflow chamber, the particles pass through the shaking and rolling movement [13]. These movements intensify the filtering and aggregation, which leads to a different packing density of the powder bed [16].

Therefore, it is important to understand the mechanisms of powder spreading, which causes a variation of the powder distribution and thus variations of the laser indentation and thermomechanical processes in Additive Manufacturing. Considering the aforementioned phenomena, this study was carried out by investigating the porosity, density, and tensile properties of specimens that were built up at two different locations. The potential of laser penetration and laser absorption as well as the thermomechanical properties that occurred during the SLM process were analyzed and interpreted in this article. $\mathrm{Co}-\mathrm{Cr}-\mathrm{W}-$ Mo alloy powder was used to fabricate the specimens, due to its high biocompatibility, ductility, and economic availability [9]. However, in order to obtain additional evidence, a widely used material, i.e., a Ti-6Al-4V alloy, was also used to fabricate test specimens and the effects of powder spreading on density are presented in this article. In summary, these specimens show the influence of powder spreading over distance, resulting in significant variations in the properties of the SLM product.

\section{Materials and methods}

\subsection{Material used}

The specimens were manufactured using fully dense $\mathrm{Co}-\mathrm{Cr}-$ W-Mo alloy and Ti-6Al-4V alloy (grade 5) powder particles which were provided by Scheftner, Germany. The diameters of the $\mathrm{Co}-\mathrm{Cr}-\mathrm{W}-\mathrm{Mo}$ alloy particles were in the range of 10 $30 \mu \mathrm{m}$ whereas the Ti-6Al-4V alloy particles' diameters were between 10 and $45 \mu \mathrm{m}$. Some tiny particles having diameters about $5 \mu \mathrm{m}$ are present in both powders. The bulk density of these alloys is $8.8 \mathrm{~g} / \mathrm{cm}^{3}$ and $4.45 \mathrm{~g} / \mathrm{cm}^{3}$, respectively. The chemical compositions of $\mathrm{Co}-\mathrm{Cr}-\mathrm{W}-\mathrm{Mo}$ alloy are $59.0 \mathrm{wt} \%$ Co, $25.0 \mathrm{wt} \% \mathrm{Cr}, 9.5 \mathrm{wt} \% \mathrm{~W}, 3.5 \mathrm{wt} \% \mathrm{Mo}$, and $1 \mathrm{wt} \% \mathrm{Si}$. Whereas, Ti-6Al-4V alloy powder contains $89 \% \mathrm{Ti}, 6 \% \mathrm{Al}$, $4 \% \mathrm{~V}$, and $<1 \%$ of the elements $\mathrm{N}, \mathrm{C}, \mathrm{H}, \mathrm{Fe}$, and $\mathrm{O}$.

\subsection{Specimen selecting and locating}

Two forms of Co-Cr-W-Mo alloy specimens were prepared as shown in Fig. 1b, using cuboidal specimens to measure density and porosity and tensile specimens to determine tensile properties. The dimensions of the cuboidal specimens were $3 \times 5 \times 8$ $\mathrm{mm}^{3}$. The tensile specimens had a gauge length of $15.34 \mathrm{~mm}$, a gauge width of $2 \mathrm{~mm}$, a thickness of $1 \mathrm{~mm}$ (continuous), a radius of $3 \mathrm{~mm}$ at the fillet, and a total length of $33 \mathrm{~mm}$. These specimens were prepared with an Arrow LMP100 SLM machine (small SLM machine) equipped with a $100-\mathrm{W}$ Yb fiber laser with a building chamber volume of $90 \times 90 \times 70 \mathrm{~mm}^{3}$ and manufactured by Dentas, LLC, Maribor, Slovenia. Therefore, the powder coating length on the build tray was $90 \mathrm{~mm}$. The specimens were placed in two positions, considering the powder coating direction, as shown in Fig. 1a by the three-dimensional schematic representation. Position 1 was chosen near the start of powder coating, approximately $10 \mathrm{~mm}$ from the coating start line. Position 2 was chosen near the end of the powder coating, between 70 and $80 \mathrm{~mm}$ from the coating start line.

To obtain further evidence, cuboidal samples of the Ti$6 \mathrm{Al}-4 \mathrm{~V}$ alloy were prepared in another SLM machine, the Arrow LMP200 (big SLM machine), equipped with a 200$\mathrm{W} \mathrm{Yb}$ fiber laser provided by Dentas, LLC, Maribor, Slovenia. The dimensions of the building chamber of this machine are $125 \mathrm{~mm}$ in the $\mathrm{X}$ direction, $125 \mathrm{~mm}$ in the $\mathrm{Y}$ direction , and $200 \mathrm{~mm}$ in the $\mathrm{Z}$ direction. Therefore, the powder coating length on the build tray was $125 \mathrm{~mm}$. In a similar way, fabricating locations 1 and 2 were selected in-between 10 and $20 \mathrm{~mm}$ and 105 and $115 \mathrm{~mm}$ from the beginning of powder coating.

\subsection{Fabricating process conditions}

The specimens of both materials were prepared at $20^{\circ} \mathrm{C}$ in a shielding gas atmosphere with an oxygen content of $0.0-0.5 \%$ in the process chamber. The powder was deposited by a recoater from one side to the other side for each layer. The laser power $70 \mathrm{~W}$, the scanning speed $800 \mathrm{~mm} / \mathrm{s}$, the track overlap $40 \%$, and the layer thickness $0.025 \mathrm{~mm}$ were selected to fabricate $\mathrm{Co}-\mathrm{Cr}-\mathrm{W}-\mathrm{Mo}$ alloy specimens. The shielding gas was nitrogen. Whereas, these parameters were $60 \mathrm{~W}, 1000 \mathrm{~mm} / \mathrm{s}$, $30 \%$, and $0.025 \mathrm{~mm}$ for the Ti- $6 \mathrm{Al}-4 \mathrm{~V}$ alloy specimens, and the shielding gas was argon. The laser focus diameter was $0.035 \mathrm{~mm}$ for the $\mathrm{Co}-\mathrm{Cr}-\mathrm{W}-\mathrm{Mo}$ alloy in the small SLM machine and $0.045 \mathrm{~mm}$ for the Ti-6Al-4V alloy in the big SLM machine. The parameters offer high productivity, while the density meets the requirements of prosthodontics. The area of the powder supply chamber was $11,370 \mathrm{~mm}^{2}$ (approx. $91 \mathrm{~mm} \times 125 \mathrm{~mm}$ ) and was increased by $0.033 \mathrm{~mm}$ for each powder recoating step.

\subsection{Density measurement}

The Archimedes' principle was applied in measuring the density of the cuboid $\mathrm{Co}-\mathrm{Cr}-\mathrm{W}-\mathrm{Mo}$ and $\mathrm{Ti}-6 \mathrm{Al}-4 \mathrm{~V}$ alloy specimens. The weights of the specimens in air and under ethanol were measured with an electronic device with an accuracy of \pm $0.1 \mathrm{mg}$. The average density of the specimens from each location was taken into account by measuring the six specimens. The maximum and minimum density measurement errors 

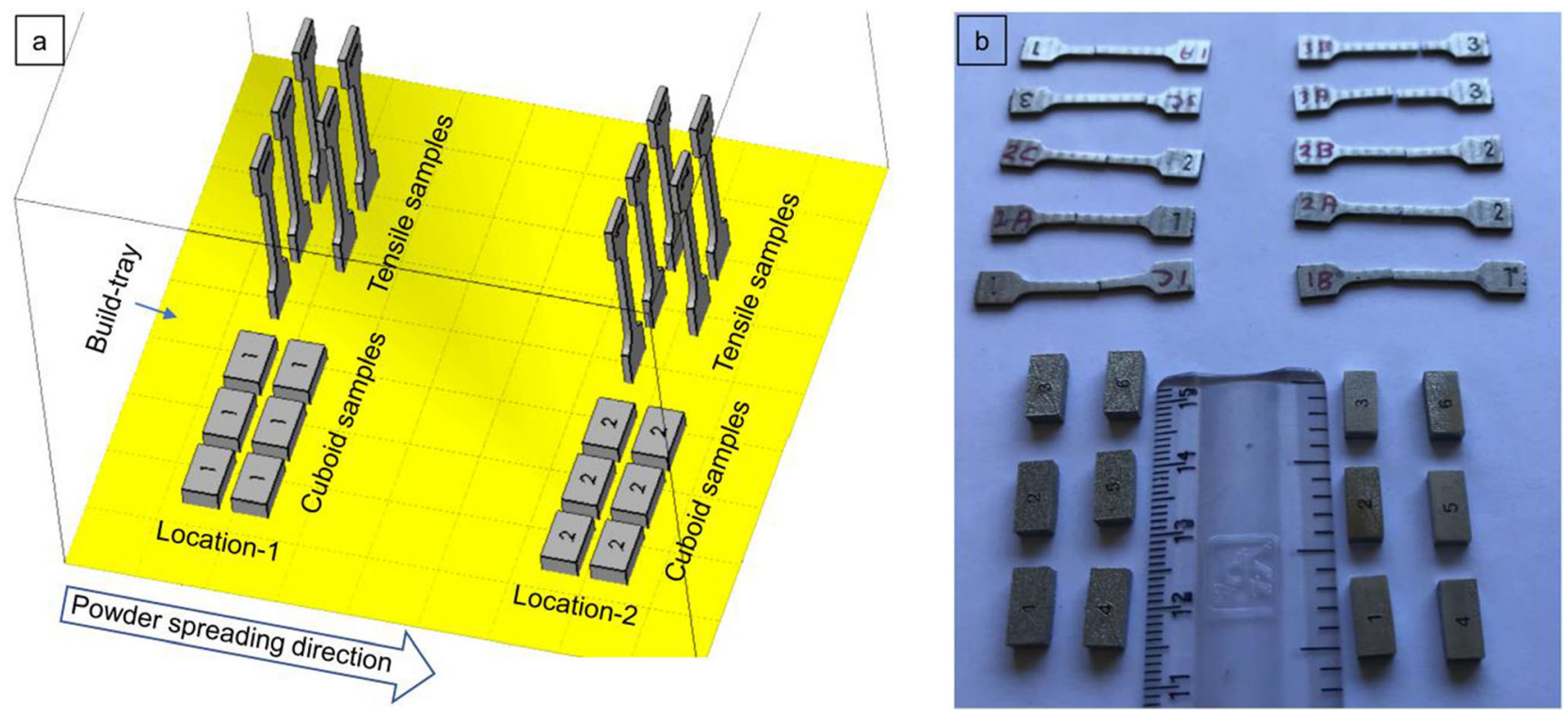

Fig. 1 Manufacturing of the specimens. a Three-dimensional schematic diagram of placing the specimens. b Manufactured specimens (the tensile specimens are after having tensile testing)

were plotted using six specimens along with their sixfold density measurements.

\subsection{Porosity determination}

The porosity was observed and measured using a computed tomography (CT) scanner, Metrotom 1500, provided by Carl Zeiss. Three of the CT-scanned images for each location were presented in this article. Only $\mathrm{Co}-\mathrm{Cr}-\mathrm{W}-\mathrm{Mo}$ alloy cuboid specimens were scanned, where all six cuboid specimens were taken into account for both locations.

\subsection{Tensile testing}

The tensile tests were performed at room temperature at a speed of $0.1 \mathrm{~mm} / \mathrm{min}$ in a Computer Console Control Panel 8500 plus with an INSTRON-1255 data acquisition system. Five tensile specimens were considered for both fabricating locations to evaluate the tensile properties. The maximum and minimum yield strength, ultimate tensile strength (UTS), and elongation were plotted on the bar graph.

\subsection{Powder packing density resolving}

After completion of the manufacturing processes, the powders were collected at two of the aforementioned locations and observed under the microscopes. The powder packing density for each location was seen using scanning electron microscope images (SEM). To see the SEM images, powder samples were collected to the adhesive tape by dipping the tape into the bulk powder. Loose particles were removed by shaking and blowing by the air stream. However, the tiny particles eventually adhered to the medium and the big particles due to the agglutination mechanism, especially the electrostatic force. It can be misconceived by observing the SEM image that some powder particles accumulated and created mounded areas, which in reality are the bumpy zones of the adhesive tape. To intensify the resolution of the different powder packing density occurrence, the powders were collected from both SLM machines for both materials after the completion of three SLM machining processes. As already mentioned, the SLM machines were Arrow LMP100 (small SLM machine) and Arrow LMP200 (big SLM machine) with a powder coating length of $90 \mathrm{~mm}$ and $125 \mathrm{~mm}$, respectively. Four images were examined for each location and for each fabrication process.

Additionally, the powder grain sizes have been measured using Scandium SEM Images software provided by Olympus, Japan. The basic interactive measurements of the distance diameter of individual spherical powder grains were done for at least 100 particles. Data were exported to MS-Excel to perform the statistical analyses. This examination assisted to count the particle size distribution and counting the numbers of tiny, small, medium, and big powder particles between two locations. Thereafter, the average ratios of each classified sized particles presence between the locations have been delivered in this article.

\section{Results}

\subsection{Density difference}

The analyses of density, porosity, and tensile properties of the specimens produced indicate that the location of the 
specimens in relation to the spreading direction of the powder has a significant influence on SLM product quality. The properties of the specimens are different at different locations on the build tray. The densities were measured for all six cuboid specimens for each location. The densities of the specimens built up near the powder spreading start line (location 1) were higher than those of the specimens built up at the location away from the powder spreading start line (location 2). Figure $2 \mathrm{a}, \mathrm{b}$ show the densities of the cuboid specimens made of the alloy $\mathrm{Co}-\mathrm{Cr}-\mathrm{W}-\mathrm{Mo}$ and the cuboid specimens made of the alloy $\mathrm{Ti}-6 \mathrm{Al}-4 \mathrm{~V}$, respectively. The $\mathrm{Co}-\mathrm{Cr}-\mathrm{W}-\mathrm{Mo}$ specimens at location 1 achieved an average density of $8.60 \mathrm{~g} / \mathrm{cm}^{3}$, while the samples at location 2 had $8.55 \mathrm{~g} / \mathrm{cm}^{3}$. Ti-6Al-4V alloy specimens gave similar results and their densities were $4.27 \mathrm{~g} / \mathrm{cm}^{3}$ at location 1 and $4.21 \mathrm{~g} / \mathrm{cm}^{3}$ at location 2 .

\subsection{Porosity detection}

Similar consequences were observed in porosity detection using CT scanning. The volumetric porosities of the cuboid specimens of the alloy $\mathrm{Co}-\mathrm{Cr}-\mathrm{W}-\mathrm{Mo}$ are shown in Fig. 3. The specimens fabricated at location 1 contain comparatively fewer pores than the specimens belonging to location 2, as shown in Fig. 3a-c and Fig. 3d-f, respectively. Moreover, it can be seen that the samples at location 2 contain a higher number of medium-sized pores than the specimens at location 1. A small number of large pores can be observed in the specimens at location 2 , which is rarely observed in the specimens fabricated at location 1. On close observation, it can also be noticed that the medium and large pores have a higher irregularity in shape. These irregularly shaped pores indicate that they were formed by moderate and large spattering during fabrication. The small pores can occur due to the small scale of spattering, collapse of keyholes, inclusion of gas bubbles, insufficient melting or lack of material in the melt pool to fill the gap. However, these pores influence the density and mechanical properties of the specimens.

\subsection{Locational effect on the tensile properties}

The effects of density, porosity, and other defects were attributed to the tensile properties of the $\mathrm{Co}-\mathrm{Cr}-\mathrm{W}-\mathrm{Mo}$ alloy specimens fabricated at two different locations. The ultimate tensile strength (UTS) of the specimens fabricated at location 2 was reduced compared to location 1 and is $954 \mathrm{MPa}$ and 980 MPa, respectively, as shown in Fig. 4a. Likewise, their yield strengths are $742 \mathrm{MPa}$ and $796 \mathrm{MPa}$, respectively, as depicted in Fig. $4 \mathrm{~b}$. The elongations at break were $4.6 \%$ and $6.5 \%$, respectively, as depicted in Fig. 4c, which also indicates the effects of the powder flow and arrangement mechanisms on the manufacturing process. It is therefore preferable, for the SLM systems that use a raking powder spreading mechanism, to build the SLM part as close as possible to the starting line of the powder coating where the diameters of the powder particles show a significant difference.

\subsection{Powder particle size distribution}

The considerable differences in powder particle size distributions between the two locations were observed. This

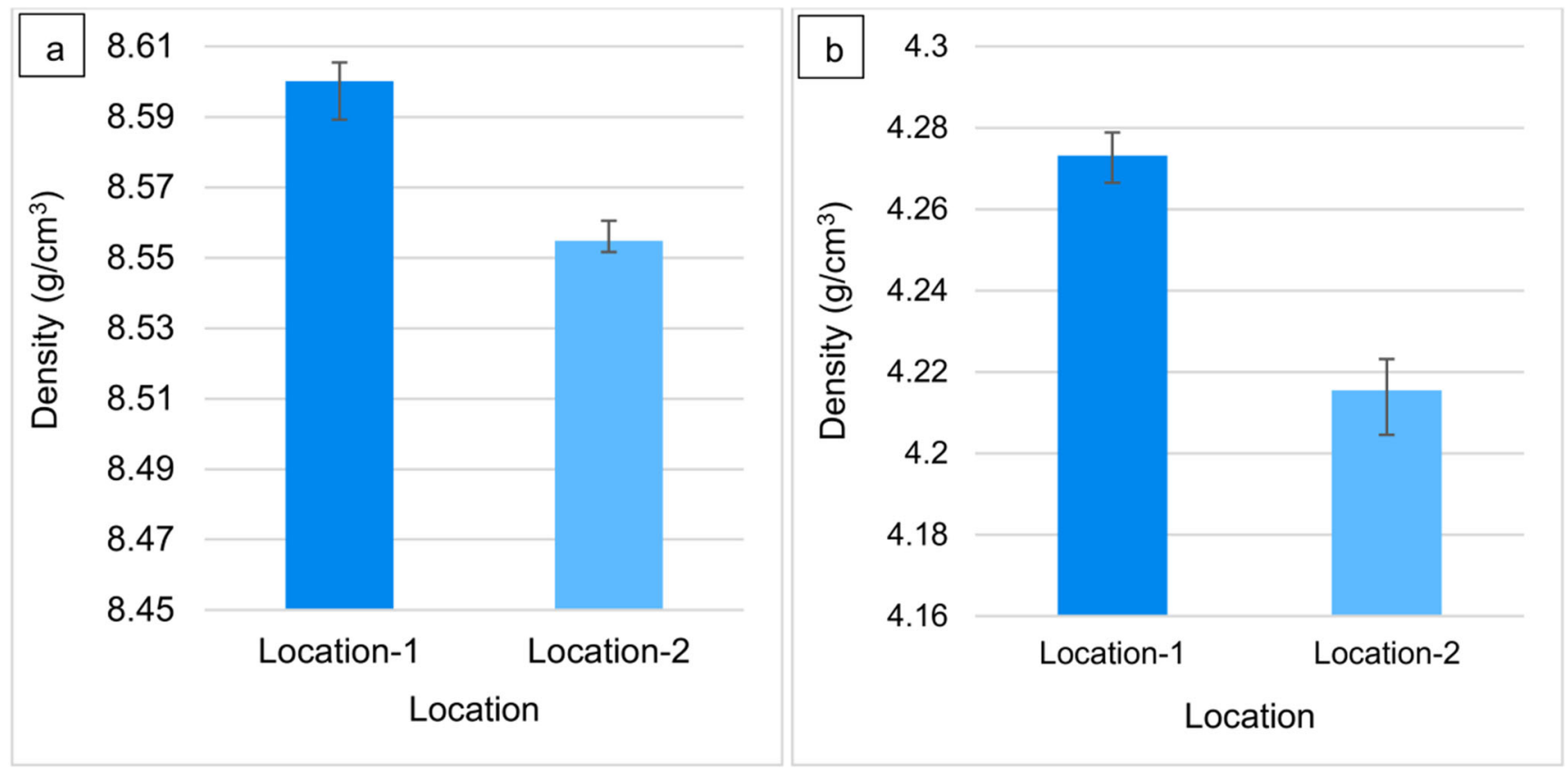

Fig. 2 Densities of the specimens built up at different locations on the build tray, where the specimens were fabricated using a Co-Cr-W-Mo alloy and b Ti-6Al-4V alloy 

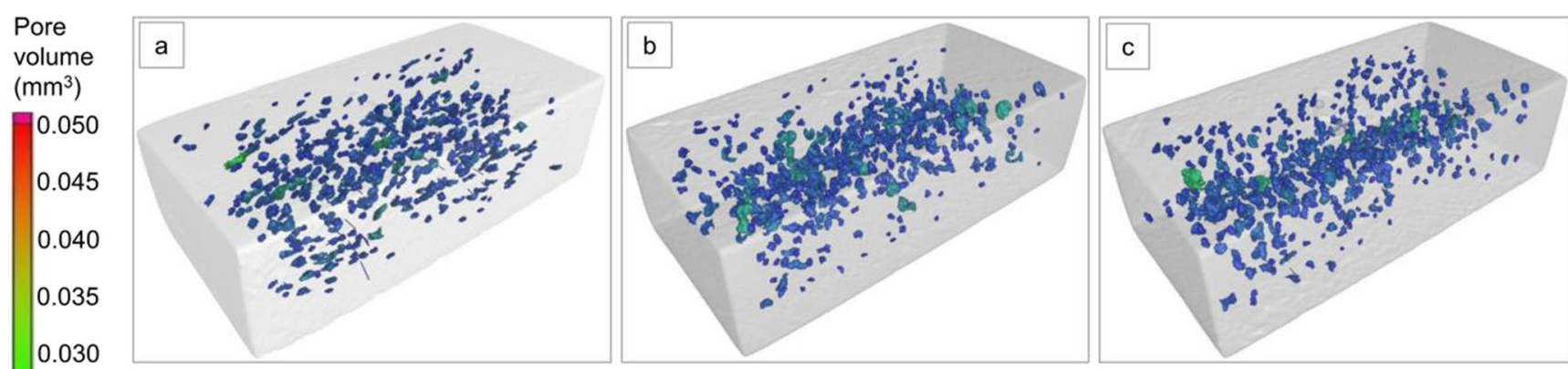

0.025
0.020
0.015
0.010
0.005
0.000
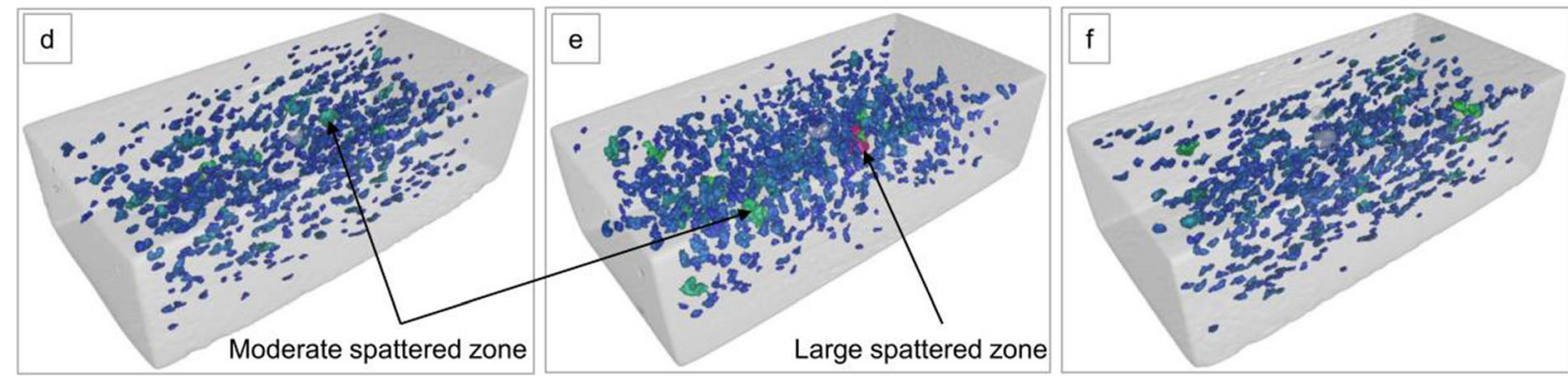

Fig. 3 The porosity of the full $\mathrm{Co}-\mathrm{Cr}-\mathrm{W}-\mathrm{Mo}$ alloy cuboid specimens, where (a)-(c) present the porosity of the specimens built up near the powder re-coater starting line (location 1) and (d)-(f) present the porosity

of the specimens built up at a place remote from the powder re-coater starting line (location 2)

phenomenon occurred for both machines as well as for both materials, as shown in Fig. 5. Figure $5 \mathrm{a}$, b shows $\mathrm{Co}-\mathrm{Cr}-\mathrm{W}-$ Mo alloy powders belonging to location 1 and location 2 respectively, collected from the small SLM machine, where the powder coating length was $90 \mathrm{~mm}$. Figure $5 \mathrm{c}$, d shows the particle distribution of $\mathrm{Co}-\mathrm{Cr}-\mathrm{W}-\mathrm{Mo}$ alloy powder samples taken from the big SLM machine at location 1 and location 2, situated at $10-20 \mathrm{~mm}$ and $105-115 \mathrm{~mm}$ from the start line, respectively. The same machine was used for the fabrication of the Ti-6Al-4V specimens after which the powder samples were taken from location 1 and location 2 . Their particle distribution is shown in Fig. 5e, f for location 1 and location 2, respectively.

A closer look at the SEM images of powder in Fig. 5 shows that location 1 contains a higher number of small and medium powder particles than location 2 , while location 2 contains a higher number of big powder particles than location 1. Even though it can be observed that location 2 contains a high
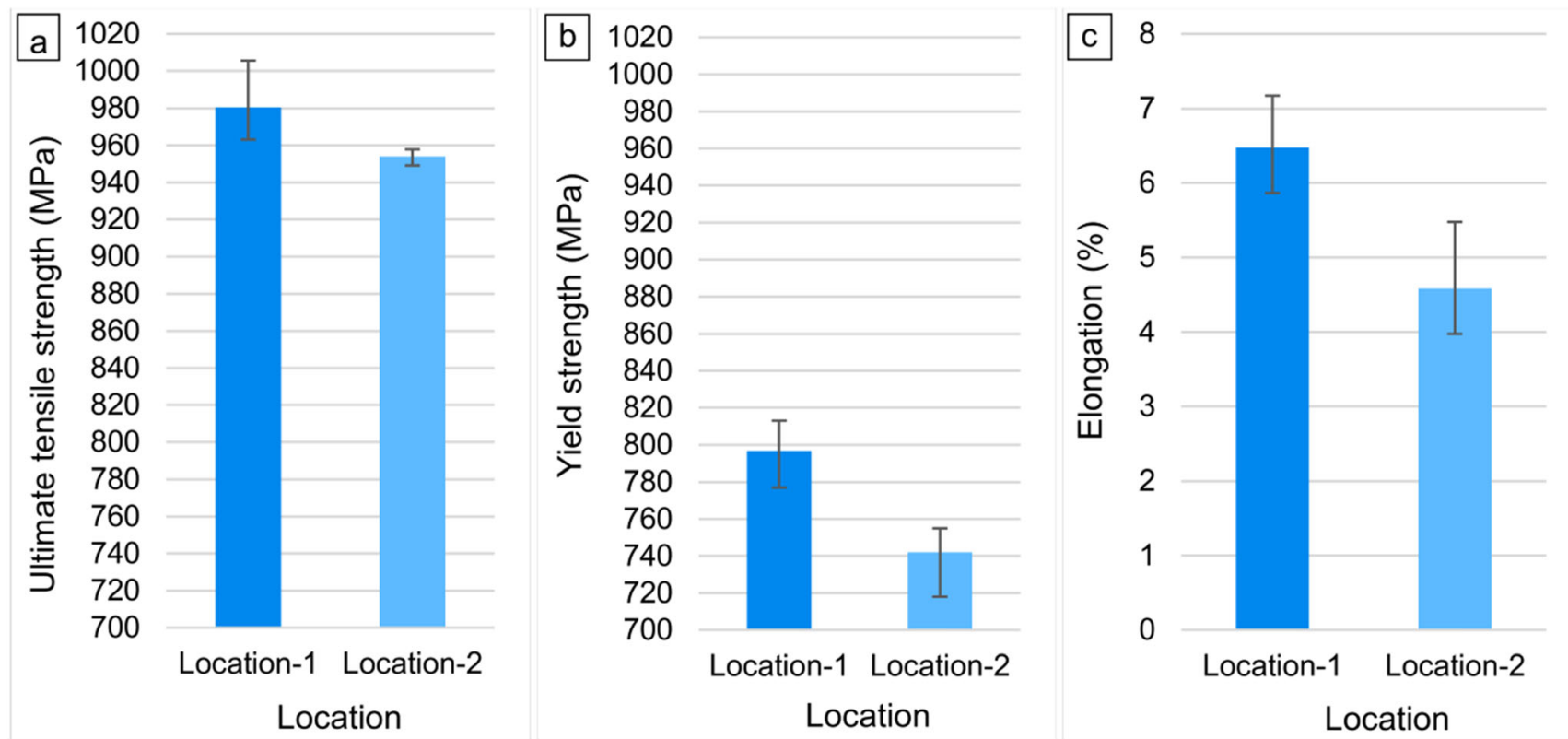

Fig. 4 Tensile properties of the Co-Cr-W-Mo alloy specimens fabricated at two different locations on the build tray, where (a) depicts ultimate tensile strengths, (b) depicts yield strengths, and (c) depicts elongations up to breaks 

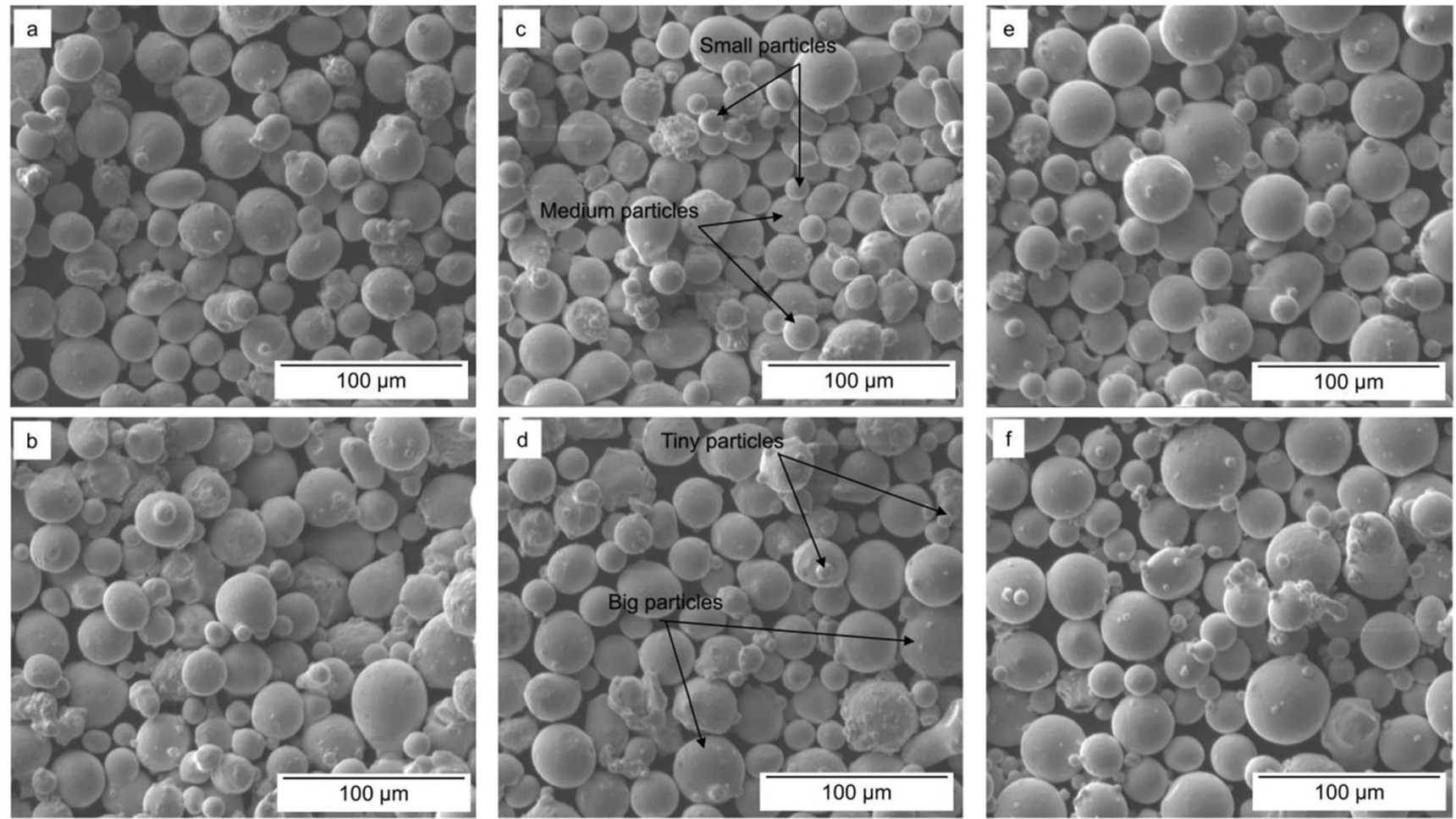

Fig. 5 SEM images of the powders collected from the different locations after finishing the fabrications in different machines, where (a) and (b) are the $\mathrm{Co}-\mathrm{Cr}-\mathrm{W}-\mathrm{Mo}$ powders belonging to location 1 and location 2 respectively collected from the small SLM machine where the powder coating length was $90 \mathrm{~mm},(\mathbf{c})$ and (d) are the $\mathrm{Co}-\mathrm{Cr}-\mathrm{W}-\mathrm{Mo}$ powders belonging to location 1 and location 2 respectively collected from the big SLM machine where the powder coating length was $120 \mathrm{~mm}$, and (e) and (f) are the Ti-6Al-4V powders belonging to location 1 and location 2 respectively collected from the big SLM machine where the powder coating length was $120 \mathrm{~mm}$ number of tiny powder particles. The diameters of the small Co-Cr-W-Mo particles are between 10 and $15 \mu \mathrm{m}$, while the medium particles are between 16 and $25 \mu \mathrm{m}$, and finally the big particles can be assumed to have a diameter above $25 \mu \mathrm{m}$. However, for both materials, some tiny particles with a diameter of about $5 \mu \mathrm{m}$ can be observed. It can be clearly observed that the particles with a diameter between 16 and $25 \mu \mathrm{m}$ are strongly represented in the powders belonging to location 1 , while the particles with a diameter above $25 \mu \mathrm{m}$ can be observed in the location 2 . For the Ti-6Al-4V alloy, the small, medium, and big particles with diameters between 10 and 20 $\mu \mathrm{m}, 21$ and $30 \mu \mathrm{m}$, and 31 and $45 \mu \mathrm{m}$ respectively can be classified. Similarly, location 1 contains a higher number of particles of Ti-6Al-4V alloy with diameters of 10-30 $\mu \mathrm{m}$ compared to location 2 . Whereas location 2 contains a higher number of bigger particles with diameters of 31-45 $\mu \mathrm{m}$.

The particle counting software provided significant consequences in powder particle size distribution. The ratio of the number of particles that existed in location 1 and location 2 are 3:1 and 3:2.4 for the particle diameters between 10 and $15 \mu \mathrm{m}$ and 16 and $25 \mu \mathrm{m}$ respectively for $\mathrm{Co}-\mathrm{Cr}-\mathrm{W}-\mathrm{Mo}$ alloy. On the other hand, this ratio became 2.2:3 for the $\mathrm{Co}-\mathrm{Cr}-\mathrm{W}-\mathrm{Mo}$ particle diameters above $25 \mu \mathrm{m}$. For the tiny particles of $\mathrm{Co}-$ $\mathrm{Cr}-\mathrm{W}-\mathrm{Mo}$, this ratio became 1:3 as expected. Similar outcomes have been observed in $\mathrm{Ti}-6 \mathrm{Al}-4 \mathrm{~V}$ alloy particle distribution as well. Their ratios are about $3: 2$ and 3:2.5 for the small and medium particles respectively considering their diameter range between 10 and $20 \mu \mathrm{m}$ and 21 and $30 \mu \mathrm{m}$, respectively. Whereas the big particles above $30 \mu \mathrm{m}$ occupied $1: 2$ in ratio. As usual, the tiny particles distributed among the location in a ratio of about 1:2. These ratios are provided taking the average number of the particle collections. However, the ratios are a little different between the SLM machines where the small and medium particles are lesser in location 2 than the location 1 in the big SLM machine where the powder coating direction was longer. Thus, the small and medium particles more dropped before reaching the location 2 . Hence, the big particles are more in number at the location 2 for this machine.

According to the SEM images of the powders belonging to both locations, it was found that the larger irregularly shaped particles are present at location 2. The shapes of the particles are very irregular, ovoid, cylindrical, and those fused from two or more particles, which is shown in Fig. 6a-d. Several particles with a very uneven surface were seen, as shown in Fig. 6b. Only very few irregular, nonspherical particles were observed in the powder belonging to location 1. Among them, most of the particles are cylindrical with smaller sizes. 

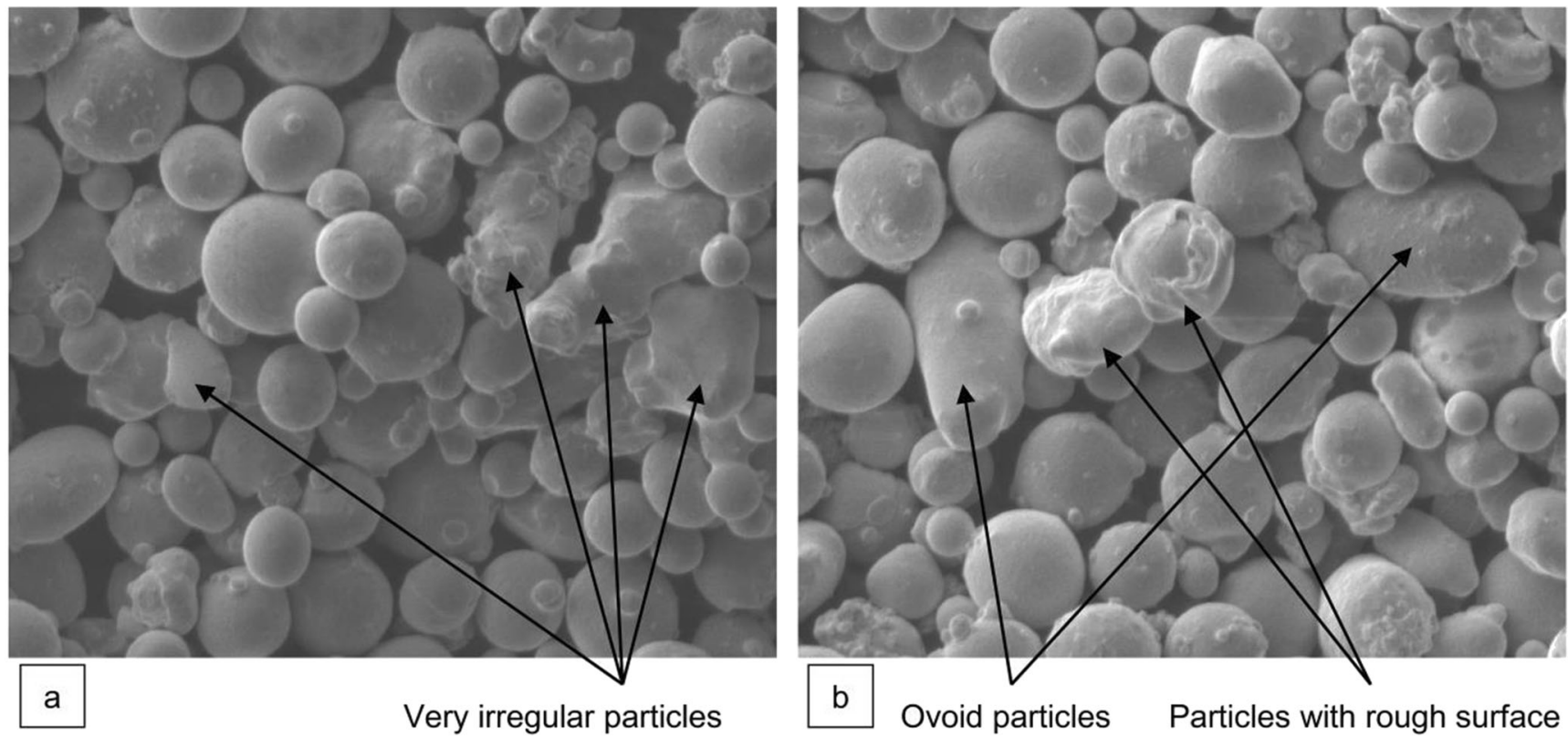

b Ovoid particles Particles with rough surface
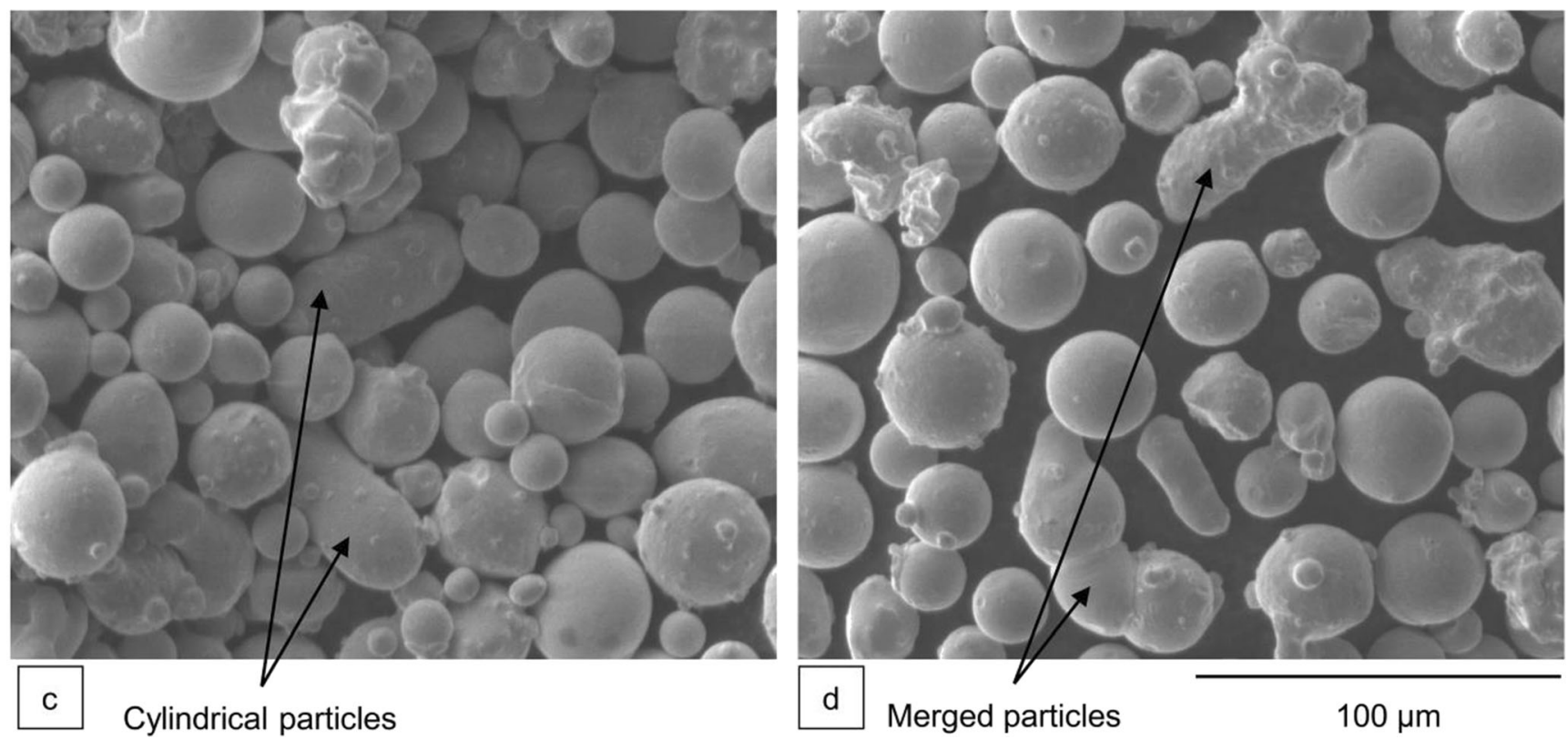

Fig. 6 Various shaped particles noticed in the $\mathrm{Co}-\mathrm{Cr}-\mathrm{W}-\mathrm{Mo}$ alloy powder belonging to location 2 where (a)-(c) were collected from the small SLM machine and (d) was collected from the big SLM machine

\section{Discussion}

\subsection{Primary cause: powder packing density}

The main cause of the consequences is a variation of the powder packing density from one location to another along the powder spreading direction. This variation was caused by the influence of the flow mechanism among different sizes of powder particles during powder layering $[16,17]$. The frictional and gravitational forces played a decisive role in the flow mechanism of the powder [13]. In addition to these forces, agglutination occurred among the particles, especially between smaller particles [18]. Surface forces such as cohesive, frictional, Van der Waals, and electrostatic forces help to agglutinate the particles [19]. Besides these mechanisms, the flowability of the powder also depends on the particle size and shape [20].

The re-coater carries a limited amount of powder with it, which decreases during the spreading. Therefore, the combined weights of the powder particles cause differences among the areas from the start to the end line of the spreading. The weight of the powder that is above the defined powder 
layer is a factor that ensures the compaction of the layer. The weight decreases from the start line to the end line of the powder spreading line, because powder is deposited and lost during spreading. Therefore, the apparent bulk density of the powder decreases along the powder spreading direction. It can therefore also be determined that the material with a higher mass causes a higher difference in the compaction of the powder. As the density of the $\mathrm{Co}-\mathrm{Cr}-\mathrm{W}-\mathrm{Mo}$ and $\mathrm{Ti}-6 \mathrm{Al}-4 \mathrm{~V}$ alloys is $8.8 \mathrm{~g} / \mathrm{mm}^{3}$ and $4.45 \mathrm{~g} / \mathrm{mm}^{3}$, respectively, the compaction difference between them was greater when considering the locations.

Moreover, the diameter of the $\mathrm{Co}-\mathrm{Cr}-\mathrm{W}-\mathrm{Mo}$ and $\mathrm{Ti}-6 \mathrm{Al}-$ $4 \mathrm{~V}$ alloy powder particles was in the range of 10 to $30 \mu \mathrm{m}$ and 5 to $45 \mu \mathrm{m}$, respectively. The particles underwent the shaking and rolling phenomenon during transfer from the powder supply tray to the overflow chamber via the building tray. The shaking results from the interactions between the particles during the forward movement by the impulsion force provided by the re-coater. The rolling is also based on this impulse force. The combined effect of the shaking and rolling phenomenon ensures that the smaller particles are filtered. This filtration is achieved by the passage of smaller particles between the interparticle gaps of the relatively bigger particles. The smaller particles moved downwards due to the gravitational force, as depicted in Fig. 7a. Eventually, the smaller particles move downwards, and likewise the bigger particles automatically move upwards by providing the gaps for the smaller particles.

Furthermore, there is a higher cohesion between the smaller particles than between the bigger particles. Therefore smaller particles adhere to each other [17]. On the other hand, the frictional force caused by rolling between adjacent particles is different for different particle sizes [13]. For the big particles, the frictional force is lower due to the reduction of the contact surfaces, which leads to an increased rotation of the powder particles. Thus, most of the smaller particles initially moved downwards and helped other small particles to move downwards and stick together by means of the Van der Waals force and the frictional force $[13,19]$. The bigger particles were easily scraped away, and usually fell onto the far end of the build tray as shown in Fig. 7b. Eventually, the bulk density of the powder bed is higher in the areas populated by the smaller particles than in the areas with bigger particles [21]. Consequently, the starting area contains a higher bulk density of the powder compared to the more distant area along the powder spreading direction.

By these aforementioned mechanisms, especially by rolling and smooth falling down, the spherical particles fall faster and most of them at their starting locations. In contrast, the irregularly shaped, nonspherical particles were scraped away and pushed to the more distant places. Hence, most of the irregularly shaped particles, such as spherical particles with high roughness (ovoid, cylindrical, etc.), can usually be observed at $\mathrm{Co}-\mathrm{Cr}-\mathrm{W}-\mathrm{Mo}$ alloy powders belonging to location 2. These particles took up more space and reduced the packing density of the powder layer.

Interestingly, although the mechanism of the smaller particles falling at the beginning of powder spreading can be observed and understood, the tiny particles are clearly visible at location 2 . The tiny particles are associated with a higher cohesive force and a lower gravitational force. Subject to these forces together with a higher frictional and electrostatic force, they cannot move downwards. Hence, they stick to the big particles and move to the distant area. An additional proof that supports the idea of the electrostatic force that occurred in the tiny particles can be drawn. It can be observed that the tiny particles adhere to the big particles, as shown in Fig. 5d, while other particles adhere to the adhesive tape. Although the excess particles were removed by shaking and blowing off during sample preparation, the tiny particles eventually adhered to the big particles. However, a higher number of tiny particles with a diameter of about $5 \mu \mathrm{m}$ can be seen on the powder sample belonging to the location 2. Eventually, they contribute advantageously to the packing density of the powder.

\subsection{Secondary cause: fusion mechanisms}

Several dissimilarities occurred in the fusion mechanisms between two different locations. The uniform compaction of the powder particles of small and medium size favors the steady penetration of the laser $[13,22]$, as shown schematically in Fig. 8a. This property supports the proper melting of the powder to create a perfect melt pool, as shown schematically in Fig. 8b. Moreover, the melt pool, supported by the surrounding particles, can obtain physical stability. These processes allow less spattering of powder particles and spattering of molten metal. Due to the higher compaction of the powder, less powder spattering occurred, and the particles were bonded together by agglutination. On the other hand, there are fewer tiny particles at location 1 because they had a higher potential to spatter from the actioning zone. Eventually, location 1 lost a small amount of material. Also, the possibility of collapsing keyholes will be less due to the constant supply of material and the uniform laser penetration at this location.

Unfavorably, bigger particles on the top of the layer reduce the penetration of the laser [23]. A high proportion of the laser beam is reflected from the top surface of the layer, as a smaller number of interparticle spaces remain, as depicted in Fig. 8c. This phenomenon leads to insufficient absorption of the thermal energy in the action zone. Insufficient heat is either unable to melt all powder particles or causes a high melt pool viscosity. Highly viscous molten metal cannot flow into the unmelted zone to fill the pores. In addition, this molten metal is easily obstructed by the big particles, which negatively affects the pore formation at location 2. Furthermore, this melt pool is not able to melt all particles perfectly as shown in Fig. 8d. 

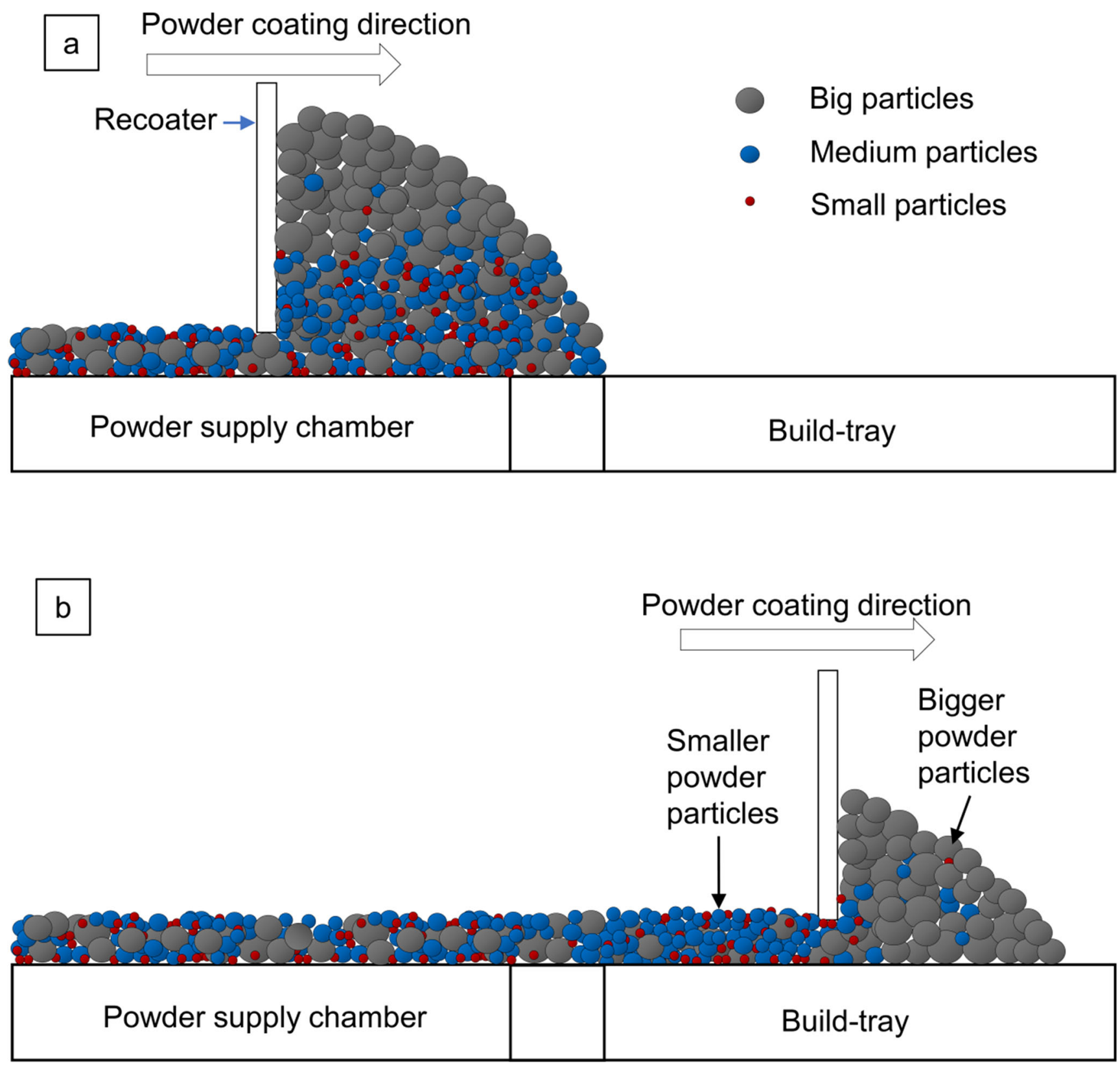

Fig. 7 Powder flow and arrangement mechanisms during collecting and spreading them during the SLM process. a The re-coater collecting the powder from the supply chamber and $\mathbf{b}$ the re-coater spreading the power on the build tray

Likewise, the melt pool penetrates poorly into the preceding layer, creating pores in the boundary between the two layers.

On the other hand, big- and medium-sized irregular particles create open spaces, which in turn results in a lower packing density. These large open spaces allow a high penetration depth of the laser, as depicted schematically in Fig. 8e. This can lead to a rapid high heat build-up during the action, which in turn causes an explosion due to a sudden expansion of the gas trapped in the interparticle spaces. This phenomenon leads to massive material spattering, through which the melt pool loses a lot of material [24]. On the other hand, a higher void space means less material in the zone. Therefore, a high induction of the laser with a small amount of material generates a small melt pool with low viscosity, as shown in Fig. 8f. A small melt pool provides space for pore formation. Since this zone absorbs high heat, it can lead to deeper melting in the preceding layer, as shown in Fig. 8f. Re-melting a large volume of the preceding layer and absorbing high energy leads to a microstructural alteration [25]. Moreover, this irregular interparticle gap interrupts the continuation of the keyhole movement and must therefore create a pore.

However, the tiny particles moved to location 2 , which had a significant positive effect on the powder packing density. Unfortunately, due to the heat shock and the low weight, these 

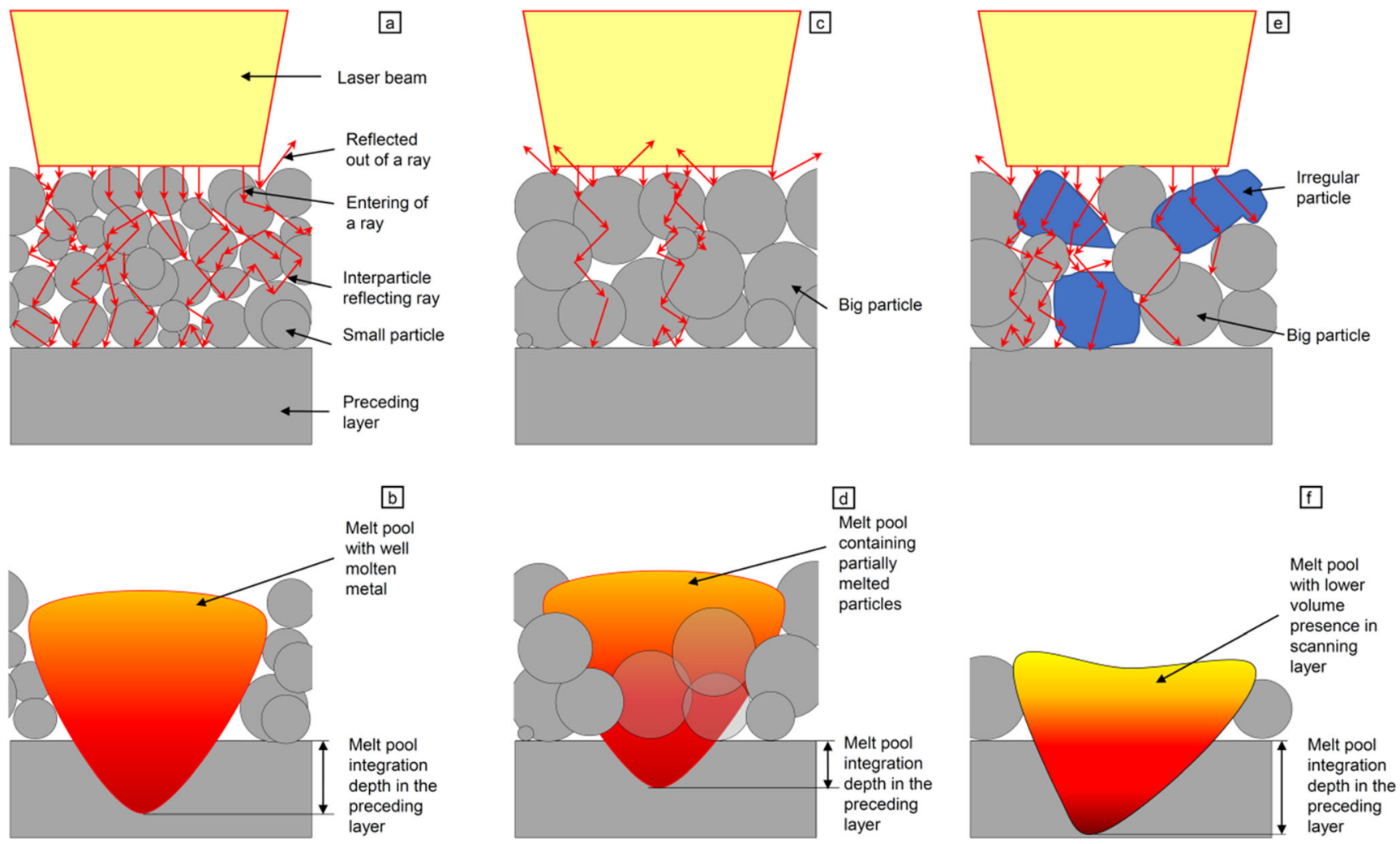

Fig. 8 Location and powder particle size-dependent melt pool formation; a schematic representation. Where (a) is the powder-laser interaction at location 1 and (b) the corresponding melt pool formation at location 1, (c) the powder-laser interaction at location 2 while particles are bigger and (d) the

corresponding melt pool formation at location 2 with partially melted particles, and (e) the powder-laser interaction at location 2 while particles are irregular and (d) the corresponding melt pool formation at location 2 with a lower level of melt pool than expected layer

particles have a high potential to spatter out of the action zone. Therefore, the tiny particles that remain on top of the layer can be removed from their positions and reduce the amount of material at the melt pool. It can also be observed that a tiny particle of Ti-6Al-4V alloy has a higher potential in the spattering mechanism than a tiny particle of $\mathrm{Co}-\mathrm{Cr}-\mathrm{W}-\mathrm{Mo}$ alloy. Overall, the $\mathrm{Co}-\mathrm{Cr}-\mathrm{W}-\mathrm{Mo}$ alloy powder at location 2 contained a higher number of irregular particles compared to Ti-6Al-4V alloy powder, but a smaller number of tiny particles were lost. These phenomena compensated for the difference in density between two different materials.

When a laser acts at the location 2, which contains big particles with a lower powder packing density, these properties can allow for higher laser penetration. However, less material creates a smaller volume of the melt pool, which gains an equal or higher amount of thermal energy than a normal melt pool. Therefore, the smaller melt pool gains a comparatively higher amount of energy, which leads to a reduction in viscosity. Due to the lower viscosity, the surface tension is also lower and material spattering increases [26]. The spattering could have occurred with molten metal or a mixture of molten metal and partially molten powder particles. Small mixed spattered particles can easily melt during subsequent scanning, while large ones remain as defects with brittleness
[27]. Therefore, places containing bigger particles absorbed an enormous amount of energy resulting in higher spatter and defects at location 2.

There is another perspective of powder particle spattering due to the heat shock and the expansion of the gas that exists at the interparticle spaces. The bigger particles get a stronger topological depression due to the rapid heating, which leads to spattering and thus to a denudation at the action zone [26]. Moreover, the bigger powder particles provide larger spaces for the inert gas. During the rapid heating, the inert gas expands rapidly and exerts a thrust on the particles, which increases the spattering of the powder particles from the surroundings of the action zone. Finally, due to their different locations along the powder spreading direction, the samples received different amounts of material, resulting in the different densities among them.

The tensile properties of the specimens were directly influenced by their density, porosity, defects, and melting mechanism. The higher number of spattering defects and pores that occurred in the specimens belong to location 2 . These defects and pores are very critical due to their brittleness under mechanical stress, and the pores are very irregular, with trenches and corners. The trenches and corners accumulate stresses and initiate microcracks, resulting in lower elongation at break. 
Higher energy caused the higher brittleness and together with the higher number of pores caused the reduction of strain in the specimens at location 2 .

\section{Conclusions}

The authors have investigated and reported the occurrence of different densities, porosities, and tensile properties of the selectively laser-melted specimens fabricated at different locations on the build tray. The densities of the specimens decreased from the start line to the end line of the powder spreading direction because of the increased porosity.

The consequences occurred due to the different compaction and packing densities of the powder particles along the powder spreading direction. The shaking, rolling, and cohesion forces encouraged the smaller powder particles to fall down near the spreading starting line. The bigger particles rolled away by means of the lower frictional force and were scraped off by the re-coater, which dropped them into remote areas.

Although the results of this research were obtained on specific SLM machines, the gained knowledge can be generalized for use in any AM powder bed system equipped with a recoater based on the raking mechanism.

Author contributions Snehashis Pal: conceptualization, methodology, visualization, writing (original draft). Nenad Gubeljak: investigation. Tonica Bončina: investigation. Radovan Hudák: investigation, funding acquisition. Teodor Toth: investigation. Jozef Zivcak: investigation. Gorazd Lojen: investigation. Niko Leben: manufacturing investigation. Igor Drstvenšek: conceptualization, supervision, writing (review and editing), funding acquisition.

Funding The authors acknowledge the financial support from the Slovenian Research Agency (Research Core Funding No. P2-0157, Research Project J1-2470 Biofunctionalization of 3D-printed metal alloys as a newly emerging strategy to diminish undesired effects of orthopedic implants, Research project J3-9262 Advanced surface finishing technologies for antibacterial properties of patient-specific 3D-printed implantable materials). This project has also received funding from the European Union's Horizon 2020 research and innovation program under grant agreement No. 788361. The research work was also supported by the projects Slovak Research and Development Agency having grant number APVV-17-0278, APVV-14-0294, and APVV-15-0356, The Ministry of Education, Science, Research and Sport of the Slovak Republic having grant number VEGA-1/0179/19, IMTS 26220220185, and ITMS2014+: 313011D103, and the incentives for research and development Stimuly BE 81 3DPTISSUE.

Data availability Nil.

\section{Compliance with ethical standards}

Competing Interests The authors declare that they have no conflict of interest.

Ethical approval The consent of this research is not involved with human and animal participants.
Consent to participate No participation is included in this research.

Consent to publish The authors agree with the copyright transfer statement.

Open Access This article is licensed under a Creative Commons Attribution 4.0 International License, which permits use, sharing, adaptation, distribution and reproduction in any medium or format, as long as you give appropriate credit to the original author(s) and the source, provide a link to the Creative Commons licence, and indicate if changes were made. The images or other third party material in this article are included in the article's Creative Commons licence, unless indicated otherwise in a credit line to the material. If material is not included in the article's Creative Commons licence and your intended use is not permitted by statutory regulation or exceeds the permitted use, you will need to obtain permission directly from the copyright holder. To view a copy of this licence, visit http://creativecommons.org/licenses/by/4.0/.

\section{References}

1. Kelly CN, Evans NT, Irvin CW et al (2019) The effect of surface topography and porosity on the tensile fatigue of $3 \mathrm{D}$ printed Ti-6Al4V fabricated by selective laser melting. Mater Sci Eng C 98:726736. https://doi.org/10.1016/j.msec.2019.01.024

2. Pal S, Kokol V, Gubeljak N et al (2019) Dimensional errors in selective laser melting products related to different orientations and processing parameters. Mater Tehnol 53:551-558. https://doi. org/10.17222/mit.2018.156

3. Demir AG, Previtali B (2017) Additive manufacturing of cardiovascular $\mathrm{CoCr}$ stents by selective laser melting. Mater Des 119: 338-350. https://doi.org/10.1016/j.matdes.2017.01.091

4. Pupo Y, Monroy KP, Ciurana J (2015) Influence of process parameters on surface quality of CoCrMo produced by selective laser melting. Int J Adv Manuf Technol 80:985-995. https://doi.org/10. 1007/s00170-015-7040-3

5. Kruth J-P, Levy G, Klocke F, Childs THC (2007) Consolidation phenomena in laser and powder-bed based layered manufacturing. CIRP Ann 56:730-759. https://doi.org/10.1016/j.cirp.2007.10.004

6. Darvish K, Chen ZW, Pasang T (2016) Reducing lack of fusion during selective laser melting of CoCrMo alloy: effect of laser power on geometrical features of tracks. Mater Des 112:357-366. https://doi.org/10.1016/j.matdes.2016.09.086

7. Lo YL, Liu BY, Tran HC (2019) Optimized hatch space selection in double-scanning track selective laser melting process. Int J Adv Manuf Technol 105:2989-3006. https://doi.org/10.1007/s00170019-04456-w

8. Pal S, Gubeljak N, Hudak R et al (2018) Tensile properties of selective laser melting products affected by building orientation and energy density. Mater Sci Eng A 743:637-647. https://doi. org/10.1016/J.MSEA.2018.11.130

9. Sidambe AT (2018) Effects of build orientation on 3d-printed cocr-mo: surface topography and 1929 fibroblast cellular response. Int J Adv Manuf Technol 99:867-880. https://doi.org/10.1007/ s00170-018-2473-0

10. Kadirgama K, Harun WSW, Tarlochan F et al (2018) Statistical and optimize of lattice structures with selective laser melting (SLM) of Ti6AL4V material. Int J Adv Manuf Technol 97:495-510. https:// doi.org/10.1007/s00170-018-1913-1

11. Stef J, Poulon-Quintin A, Redjaimia A et al (2018) Mechanism of porosity formation and influence on mechanical properties in selective laser melting of Ti-6Al-4V parts. Mater Des 156:480-493. https://doi.org/10.1016/j.matdes.2018.06.049 
12. Simonelli M, Tse YY, Tuck C (2014) Effect of the build orientation on the mechanical properties and fracture modes of SLM Ti-6Al4V. Mater Sci Eng A 616:1-11. https://doi.org/10.1016/j.msea. 2014.07.086

13. Gu D, Xia M, Dai D (2019) On the role of powder flow behavior in fluid thermodynamics and laser processability of Ni-based composites by selective laser melting. Int J Mach Tools Manuf 137:67-78. https://doi.org/10.1016/j.ijmachtools.2018.10.006

14. Nagarajan B, Hu Z, Song X et al (2019) Development of micro selective laser melting: the state of the art and future perspectives. Engineering 5:702-720. https://doi.org/10.1016/j.eng.2019.07.002

15. Chen H, Wei Q, Wen S et al (2017) Flow behavior of powder particles in layering process of selective laser melting: numerical modeling and experimental verification based on discrete element method. Int J Mach Tools Manuf 123:146-159. https://doi.org/10. 1016/j.ijmachtools.2017.08.004

16. Yao D, An X, Fu H et al (2020) Dynamic investigation on the powder spreading during selective laser melting additive manufacturing. Addit Manuf:101707. https://doi.org/10.1016/j. addma.2020.101707

17. Wang L, Li EL, Shen $\mathrm{H}$ et al (2020) Adhesion effects on spreading of metal powders in selective laser melting. Powder Technol 363: 602-610. https://doi.org/10.1016/j.powtec.2019.12.048

18. Taguchi I, Kurashige M, Imai K (2006) Effects of cubic container's wall or floor on random packing structures of spherical particles. JSME Int J Ser A Solid Mech Mater Eng 49(2):265-272. https:// doi.org/10.1299/jsmea.49.265

19. Lu K, Reynolds WT (2008) 3DP process for fine mesh structure printing. Powder Technol 187:11-18. https://doi.org/10.1016/j. powtec.2007.12.017

20. Alyami H, Dahmash E, Bowen J, Mohammed AR (2017) An investigation into the effects of excipient particle size, blending techniques \& processing parameters on the homogeneity \& content uniformity of a blend containing low-dose model drug. PLoS One. https://doi.org/10.1371/journal.pone.0178772
21. Sing SL, Yeong WY, Wiria FE et al (2017) Direct selective laser sintering and melting of ceramics: a review. Rapid Prototyp J 23(3): 611-623. https://doi.org/10.1108/RPJ-11-2015-0178

22. Zhang B, Coddet C (2016) Selective laser melting of iron powder: observation of melting mechanism and densification behavior via point-track-surface-part research. J Manuf Sci Eng Trans ASME 138(5):051001. https://doi.org/10.1115/1.4031366

23. Kovalev OB, Gusarov AV, Belyaev VV (2020) Morphology of random packing of micro-particles and its effect on the absorption of laser radiation during selective melting of powders. Int J Eng Sci 157:103378. https://doi.org/10.1016/j.ijengsci.2020.103378

24. Nakamura H, Kawahito Y, Nishimoto K, Katayama S (2015) Elucidation of melt flows and spatter formation mechanisms during high power laser welding of pure titanium. J Laser Appl 27:032012. https://doi.org/10.2351/1.4922383

25. Li K, Mao X, Khanlari K et al (2020) Effects of powder size distribution on the microstructural and mechanical properties of a Co$\mathrm{Cr}-\mathrm{W}-\mathrm{Si}$ alloy fabricated by selective laser melting. J Alloys Compd 825:153973. https://doi.org/10.1016/j.jallcom.2020. 153973

26. Khairallah SA, Anderson AT, Rubenchik A, King WE (2016) Laser powder-bed fusion additive manufacturing: physics of complex melt flow and formation mechanisms of pores, spatter, and denudation zones. Acta Mater 108:36-45. https://doi.org/10.1016/ j.actamat.2016.02.014

27. Wang D, Wu S, Fu F et al (2017) Mechanisms and characteristics of spatter generation in SLM processing and its effect on the properties. Mater Des 117:121-130. https://doi.org/10.1016/j.matdes. 2016.12.060

Publisher's note Springer Nature remains neutral with regard to jurisdictional claims in published maps and institutional affiliations. 